\section{BRAZIULIAN JOURNAL \\ OF MEDICAL AND BIOLOGICAL RESF.ARCH}

www.bjournal.com.br
ISSN 0100-879X

Volume 42 (10) 870-992 October 2009

BIOMEDICAL SCIENCES

AND

CLINICAL INVESTIGATION

Braz J Med Biol Res, October 2009, Volume 42(10) 958-962

Does hepatocellular carcinoma in non-alcoholic steatohepatitis exist in cirrhotic and non-cirrhotic patients?

A.L. Chagas, L.O.O. Kikuchi, C.P.M.S. Oliveira, D.C.P. Vezozzo, E.S. Mello, A.C. Oliveira,

L.C. Cella, P. Herman, T. Bachella, S.H. Caldwell, V.A.F. Alves and F.J. Carrilho

The Brazilian Journal of Medical and Biological Research is partially financed by

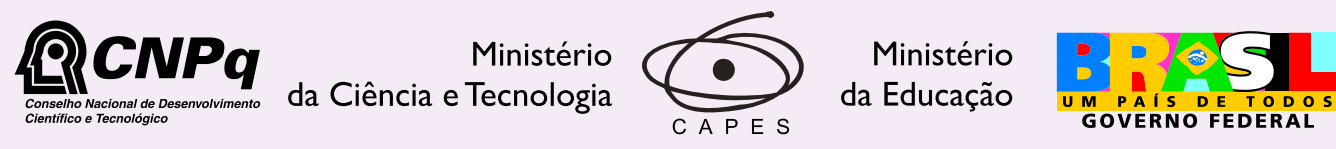

DFAPESP

Institutional Sponsors 


\title{
Does hepatocellular carcinoma in non-alcoholic steatohepatitis exist in cirrhotic and non-cirrhotic patients?
}

\author{
A.L. Chagas ${ }^{1}$, L.O.O. Kikuchi ${ }^{1}$, C.P.M.S. Oliveira ${ }^{1}$, D.C.P. Vezozzo', \\ E.S. Mello², A.C. Oliveira' ${ }^{1}$, L.C. Cella ${ }^{1}$, P. Herman ${ }^{1}$, T. Bachella1, \\ S.H. Caldwell ${ }^{3}$, V.A.F. Alves ${ }^{2}$ and F.J. Carrilho ${ }^{1}$ \\ ${ }^{1}$ Departamento de Gastroenterologia (LIM 07/37), ²Departamento de Patologia (LIM 14), \\ Faculdade de Medicina, Universidade de São Paulo, São Paulo, SP, Brasil \\ ${ }^{3}$ Department of Gastroenterology and Hepatology, University of Virginia, Charlottesville, VA, USA
}

\begin{abstract}
Non-alcoholic steatohepatitis (NASH) has been associated with hepatocellular carcinoma (HCC) often arising in histologically advanced disease when steatohepatitis is not active (cryptogenic cirrhosis). Our objective was to characterize patients with HCC and active, histologically defined steatohepatitis. Among 394 patients with HCC detected by ultrasound imaging over 8 years and staged by the Barcelona Clinic Liver Cancer (BCLC) criteria, we identified 7 cases $(1.7 \%)$ with HCC occurring in the setting of active biopsy-proven NASH. All were negative for other liver diseases such as hepatitis $\mathrm{C}$, hepatitis $\mathrm{B}$, autoimmune hepatitis, Wilson disease, and hemochromatosis. The patients (4 males and 3 females, age $63 \pm 13$ years) were either overweight (4) or obese (3); $57 \%$ were diabetic and $28.5 \%$ had dyslipidemia. Cirrhosis was present in 6 of 7 patients, but 1 patient had welldifferentiated HCC in the setting of NASH without cirrhosis (fibrosis stage 1) based on repeated liver biopsies, the absence of portal hypertension by clinical and radiographic evaluations and by direct surgical inspection. Among the cirrhotic patients, $71.4 \%$ were clinically staged as Child $A$ and $14.2 \%$ as Child $B$. Tumor size ranged from 1.0 to $5.2 \mathrm{~cm}$ and 5 of 7 patients were classified as early stage; $46 \%$ of all nodules were hyper-echoic and $57 \%$ were $<3 \mathrm{~cm}$. HCC was well differentiated in $1 / 6$ and moderately differentiated in $5 / 6$. Alpha-fetoprotein was $<100 \mathrm{ng} / \mathrm{mL}$ in all patients. $\mathrm{HCC}$ in patients with active steatohepatitis is often multifocal, may precede clinically advanced disease and occurs without diagnostic levels of alpha-fetoprotein. Importantly, $\mathrm{HCC}$ may occur in NASH in the absence of cirrhosis. More aggressive screening of NASH patients may be warranted.
\end{abstract}

Key words: Hepatocellular carcinoma; Liver cancer; Fatty liver; Steatohepatitis; Cirrhosis

\section{Introduction}

Non-alcoholic fatty liver disease (NAFLD) is a clinical and pathological condition whose spectrum can range from steatosis to steatohepatitis and cirrhosis in patients without a history of alcohol abuse (1). Non-alcoholic steatohepatitis (NASH), the severe form of NAFLD, has emerged as a clinically important type of chronic liver disease characterized pathologically by hepatocellular ballooning, Mallory's hyaline, scattered inflammation, and perisinusoidal fibrosis (2). NASH associated with cirrhosis progresses to hepatocellular cancer and reoccurs post-transplantation $(3,4)$. This disorder is now considered to be the major cause of cryptogenic cirrhosis in many regions of the world (5).

More recently, NASH has been increasingly associated with hepatocellular carcinoma ( $\mathrm{HCC})$, often arising in the late stage of the disease with non-specific histology ascribed to cryptogenic cirrhosis (6). Thus, NASH-related HCC is often thought to occur primarily in patients with advanced disease, when hepatocellular ballooning, inflammation and steatosis (e.g., NASH) have subsided. For example, Marrero et al. (7) studied the etiology of liver disease in 150 patients with HCC wherein NAFLD-related cryptogenic cirrhosis accounted for at least $13 \%$ of the cases. However, fibrosis progression in NAFLD appears to be typically slow $(8,9)$ and the clinical features of $\mathrm{HCC}$ arising in earlier stages of NASH are not well-defined, although obesity is a recognized risk factor for both NASH and HCC (10) and sporadic cases of HCC arising from non-cirrhotic NAFLD have been reported (11). The aim of this study was to characterize patients with HCC 
and histologically defined NASH with or without cirrhosis.

\section{Patients and Methods}

\section{Patients}

Between April 1998 and August 2006, 394 consecutive patients with $\mathrm{HCC}$ were evaluated at a single center (Department of Gastroenterology of Clinic Hospital of University of São Paulo School of Medicine). According to etiology, hepatitis $C$ virus was the most frequent in $58 \%$, followed by hepatitis B virus in 16\%, alcohol in $10 \%$ and cryptogenic disease in $9 \%$. We identified 7 patients $(1.7 \%)$ with histologically proven NASH. Six of 7 associated hepatocellular cancers were confirmed histologically. The protocol was approved by the Internal Review Board of the University of São Paulo (São Paulo, SP, Brazil).

\section{Diagnostic criteria}

The diagnosis of NASH was based on the following criteria: 1) a liver biopsy specimen showing steatosis ( $>10 \%$ of hepatocytes), hepatocellular ballooning and pericellular fibrosis as assessed by a liver pathologist with expertise in NAFLD; 2) the exclusion of liver diseases such as hepatitis $B$, hepatitis $\mathrm{C}$, autoimmune hepatitis, alpha1-antitrypsin deficiency, Wilson disease, and hemochromatosis; 3) the exclusion of patients who had $a>100 \mathrm{~g} /$ week alcohol intake determined by a detailed personal history, questioning of family members, and an investigation of previous medical records, and 4) the exclusion of patients with steatohepatitis accompanying other liver diseases, or systemic diseases other than obesity, hyperlipidemia, and diabetes or with an intake of hepatotoxic drugs or lipid-lowering agents. HCC diagnostic criteria included coincident findings by at least two imaging techniques showing characteristic features in a focal hepatic lesion $>2 \mathrm{~cm}$, with arterial hypervascularization (Barcelona Clinic Liver Cancer (BCLC) criteria) (12) and histologically confirmed in 6 of 7 patients.

\section{Laboratory assays}

Laboratory examinations included tests for hepatitis $B$ surface antigen, hepatitis B surface antibody (anti-HBs), hepatitis B core antibody (anti-HBc) by enzyme immunoassay (Dinabot, Japan), HCV antibody by third-generation enzyme immunoassay (Ortho Diagnostics, Japan), HCVRNA by the polymerase chain reaction (Amplicor HCV, Roche Diagnostic Systems, Japan), anti-nuclear antibody and anti-smooth muscle antibody by immunofluorescence on Hep 2 cells. Tests for alpha-fetoprotein (AFP) were done by EIA. Aminotransferase studies and other routine tests were performed by standard methods using automated techniques (Modular P800, Roche/Hitachi, Switzerland).

\section{Histology}

The liver tissue was fixed in $4 \%$ formaldehyde and processed for hematoxylin-eosin, Masson trichrome and picrosyrius red stains, Gomori's silver impregnation for extracellular matrix and Perls staining for pigments. All specimens were scored blindly for individual histological parameters by a liver pathologist with expertise in NAFLD: macro- and microvacuolar fatty change, zonal distribution, foci of necrosis, portal and perivenular fibrosis, and inflammatory and fibrotic infiltrate with zonal distribution and the composite NASH activity score were calculated (13). Fibrosis was assessed using a 4-grade scale. In this scheme, F1 denotes foci of perivenular/pericellular fibrosis in zone 3, F2 represents the changes defining F1 plus focal or extensive periportal fibrosis, F3 denotes bridging fibrosis (focal or widespread), and F4 indicates the presence of cirrhosis. The grade of tumor differentiation was assessed as best differentiated (grade I), well differentiated (grade II), moderately differentiated (grade III), or poorly differentiated (grade IV), according to the Edmonson \& Steiner classification.

\section{Results}

There were 4 males and 3 females with a mean age of $63 \pm 13.9$ years. Four patients $(5 \%)$ were overweight (BMI $\left.25-29.9 \mathrm{~kg} / \mathrm{m}^{2}\right)$ and 3 were obese (BMI $\left.\geq 30\right)(43 \%) ; 57 \%$ had type II diabetes and $28.5 \%$ had dyslipidemia. Cirrhosis was present in 6 of the 7 patients (Figure 1A) and 1 had HCC and NASH without cirrhosis, with a liver biopsy showing only perivenular and pericellular fibrosis (stage 1; Figure $1 \mathrm{~B}, \mathrm{C})$. Clinical and radiologic parameters confirmed the absence of evidence of portal hypertension in this patient (see details below). Among the cirrhotic patients, 71.4\% were clinically staged as Child A and $14.2 \%$ as Child B. Lesions were visualized by ultrasound in all patients. These data are summarized in Table 1.

Four patients had only one HCC nodule, 1 patient had 2 tumors, 1 patient had 3 tumors, and 1 had 4 tumors (Table 1). Tumor size ranged from 1.0 to $5.2 \mathrm{~cm}$ and 8 of 14 nodules (57\%) were $<3 \mathrm{~cm}$. Most lesions (46\%) were hyper-echoic by ultrasound. The HCC was well differentiated in $2 / 6(33.3 \%$; Figure 1D,E), moderately differentiated in 4/6 (66.6\%; Figure 1F) and poorly differentiated in none. An interesting finding was the presence of histological features of NASH in 4 of 7 cases. In several neoplastic cells, we observed focal to moderate steatosis (Figure 1E,F), ballooning and Mallory bodies (Figure 1F). In 2 cases, we found also peliosis. These intracellular lesions were apparently associated with milder histological grades. AFP was $<100$ $\mathrm{ng} / \mathrm{mL}$ in all patients (Table 1 ).

\section{HCC in NAFLD in the absence of cirrhosis}

A 65-year-old asymptomatic male was found to have increased aminotransferase values during his annual physical examination. Ultrasound revealed two liver nodules. He had a history of hypertension, hyperlipidemia and hyperuricemia. His medical history was otherwise unremarkable. He was overweight (BMI, 27.6/m²) without signs of chronic 

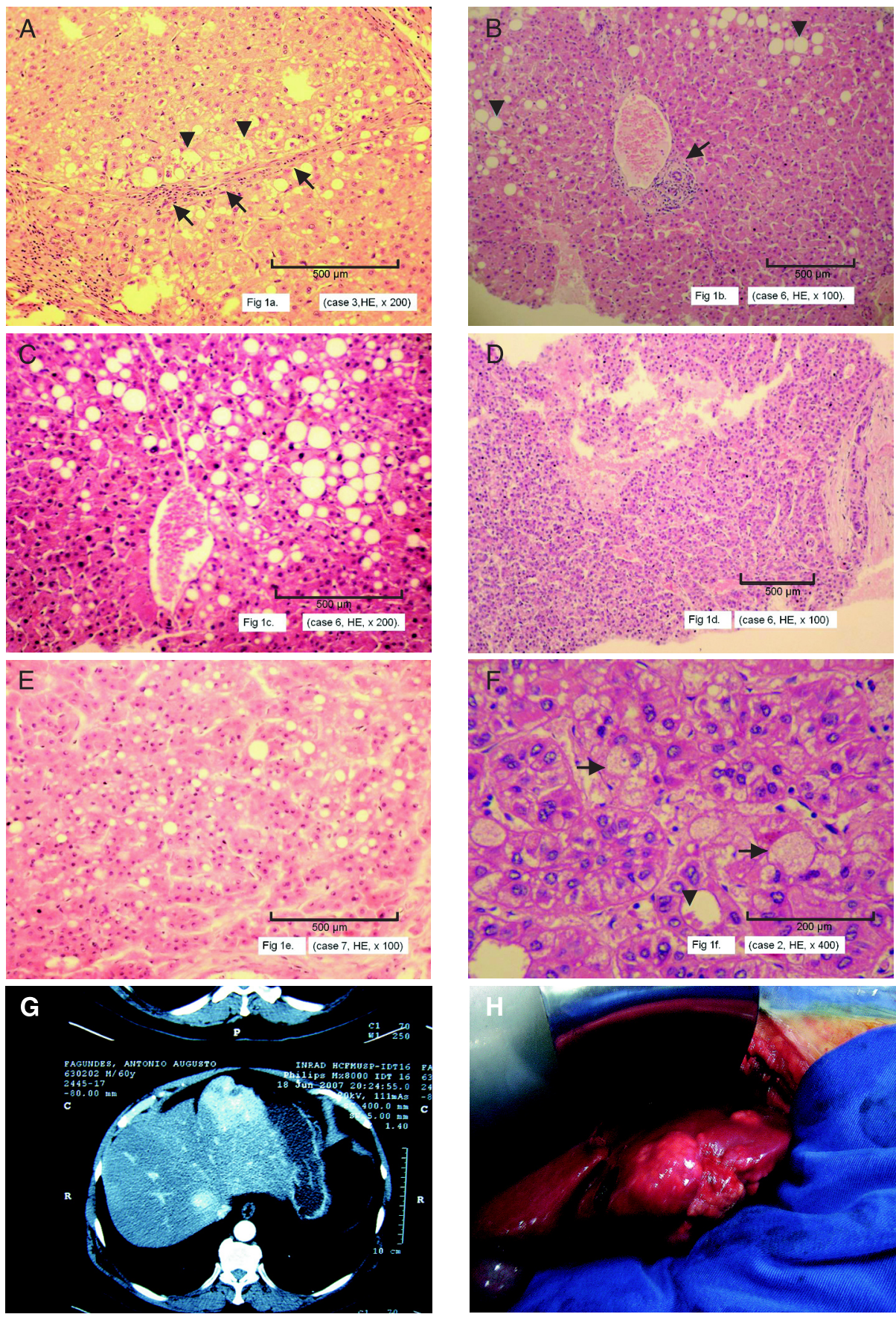

Figure 1. Microscopic features of non-neoplastic areas and of hepatocellular carcinoma (HCC). A, Hepatic cirrhosis with thin septae (arrows). Prominent hepatocellular ballooning (arrowheads) is seen together with Mallory bodies. Grade I steatosis and scarce polymorphonuclear and lymphocytic inflammation (case 3, HE, 200X). B, Steatohepatitis stage 1 contralateral to HCC. An individualized portal tract shows minimal inflammation and no fibrosis (arrow). Steatosis is seen in 15-20\% of hepatocytes (arrowheads, case 6 , HE, 100X). $C$, Another microscopic field of the same case, presenting a preserved terminal (centrilobular) venule, surrounded by steatotic hepatocytes. Some polymorphonuclears are found (case 6, HE, 200X). D, Well-differentiated HCC presenting small hepatocytes crowded in thin trabeculae in the non-alcoholic steatohepatitis (NASH) case without cirrhosis (case 6, HE, 100X). E, Well-differentiated HCC showing micro- and macrogoticular steatosis (case 7, HE, 100X). F, Moderately differentiated HCC presenting features of NASH: ballooning (arrows) and Mallory bodies (arrowhead) are found in neoplastic cells (case 2, HE, 400X). G, Computed tomographic scan indicating a steatotic liver with a hypervascular mass in segment 3. $\mathrm{H}$, Intraoperative view of a non-cirrhotic liver with a tumor located in segment 3. 
Table 1. Demographic, clinical, biochemical, and histological parameters of the 7 patients studied.

\begin{tabular}{|c|c|c|c|c|c|c|c|}
\hline & 1 & 2 & 3 & 4 & 5 & 6 & 7 \\
\hline Gender & $\mathrm{F}$ & M & $M$ & M & $\mathrm{F}$ & M & $\mathrm{F}$ \\
\hline Age (years) & 59 & 71 & 61 & 77 & 35 & 65 & 73 \\
\hline Diabetes mellitus & $\mathrm{Y}$ & $Y$ & $\mathrm{~N}$ & $\mathrm{~N}$ & $\mathrm{Y}$ & $\mathrm{N}$ & $Y$ \\
\hline Overweight & $\mathrm{Y}$ & $\mathrm{Y}$ & $\mathrm{Y}$ & Y & Y & $\mathrm{Y}$ & Y \\
\hline Dyslipidemia & $\mathrm{N}$ & $\mathrm{Y}$ & $\mathrm{N}$ & $\mathrm{N}$ & $\mathrm{N}$ & $\mathrm{Y}$ & $\mathrm{N}$ \\
\hline Cirrhosis & $\mathrm{Y}$ & $\mathrm{Y}$ & $\mathrm{Y}$ & $\mathrm{Y}$ & $\mathrm{Y}$ & $\mathrm{N}$ & $\mathrm{Y}$ \\
\hline Child-Pugh & A6 & A5 & A5 & A5 & B9 & - & A6 \\
\hline AFP (ng/mL) & 6 & 10 & 21 & 10 & 3 & 7 & 17 \\
\hline HCC Rx & TACE & Resection & $P E I+T X$ & Resection & TACE & TACE & PEI \\
\hline HCC differentiation & NA & GII & GII & GIII & NA & GI & $\mathrm{GI}$ \\
\hline Number of nodules & 1 & 1 & 3 & 1 & 4 & 2 & 1 \\
\hline Echo pattern & High & High & High & NA & Low & Mixed & High \\
\hline HCC size $(\mathrm{mm})$ & 33 & 34 & 30 & 43 & 28 & 52 & 33 \\
\hline Stage (BCLC) & Early & Early & Early & Early & Intermediate & Intermediate & Early \\
\hline
\end{tabular}

AFP = alpha-fetoprotein; HCC = hepatocellular carcinoma; TACE = transcatheter arterial chemoembolization; PEI + TX = percutaneous ethanol instillation + liver transplantation; NA = not available by ultrasound; GI, II, III = grades I, II and III, respectively; $\mathrm{BCLC}=$ Barcelona Clinic Liver Cancer criteria.

liver disease. Using ultrasound, computed tomography, and magnetic resonance imaging, we detected two hypoechoic tumors measuring $52 \mathrm{~mm}$ in the greatest dimension in segment II and $10 \mathrm{~mm}$ in segment $\mathrm{V}$, and steatosis in the background, without signs of cirrhosis or portal hypertension (Figure 1G,H). An ultrasound-guided biopsy from the largest nodule showed a grade-I trabecular HCC (Figure 1D). The non-cancerous areas showed NASH stage 1 , characterized by minimal perivenular fibrosis, moderate steatosis with mild hepatocellular ballooning and mild necroinflammation (Figure 1C). Portal tracts were normal-sized, without fibrous septa (Figure 1B). Therefore, surgical resection (left hepatectomy) for the segment 2 lesion was indicated and at the time of surgery radioablation of the tumor in segment $\checkmark$ was planned. However, since intra-operatory ultrasound showed 12 nodules, both in the right and in left hepatic lobes, it was decided not to do any hepatic resection or radioablation. The macroscopic liver aspect on surgery revealed only steatosis, without signs of cirrhosis or portal hypertension. The patient was therefore intra-operatively submitted again to a liver and tumor biopsy.

The histological findings confirmed a diagnosis of grade I $\mathrm{HCC}$ and underlying NASH stage 1 , without cirrhosis. The patient was treated with transcatheter arterial chemoembolization. $\mathrm{He}$ is alive without symptoms, 24 months after diagnosis.

\section{Discussion}

Hepatocellular carcinoma is a well-recognized end-point of the progression of $\mathrm{NASH}$ although the exact risk remains to be defined. In recent years, NASH has been proposed as an important cause of $\mathrm{HCC}$ especially in industrialized countries where obesity rates and associated fatty liver disease have become a common problem. However, most cases have been found in the setting of cryptogenic cirrhosis thought to be due to progression of NASH. In the present paper, we describe 7 cases of HCC in histologically defined $\mathrm{NASH}$ patients including one case without cirrhosis.

The clinical features of NASH patients with $\mathrm{HCC}$ studied herein differ in some respects from those of $\mathrm{HCC}$ patients with cirrhosis associated with HCV or HBV infection. In the series of 93 cirrhotic patients who developed HCC during the screening program in our group (Campos-Cella LT, Paranagua-Vezozzo DC, Farias AQ, Ono-Nita SK, Matielo $\mathrm{CH}$, Alves VAF, et al., unpublished data), we found that 57 years was the average age of patients with HCC. In the present study of NASH patients, the mean age was considerably higher with an average of 63 years, similar to a previous study reporting later HCC development in patients with NASH (14).

The most important factors for the development of HCC in most literature reports were hepatitis B and C infections. In the present series, viruses were ruled out in patients with a background of overweight in all cases, $57 \%$ of the patients had non-insulin-dependent diabetes mellitus and $28.5 \%$ had dyslipidemia. Obesity itself is a recognized risk factor for both NASH and HCC (10) and isolated cases of HCC arising from non-cirrhotic NAFLD have been reported (11). In our series, there was 1 patient of 7 with non-cirrhotic NASH who was obese.

The histological features of NASH complicated by HCC were somewhat different from $\mathrm{HCC}$ associated with other cirrhotic causes. The livers were cirrhotic in 6 patients and non-cirrhotic in 1 patient and the histological characteristics 
of HCC showed a predominance of well to moderately differentiated $\mathrm{HCC}$, similar to previously reported data regarding $\mathrm{NASH}$-related HCC (15). No case of undifferentiated HCC was detected. Intriguingly, morphologic features of NASH such as fatty change, ballooning and Mallory bodies were observed in neoplastic hepatocytes, a fact that must be further studied in prospective series. All patients studied herein had been selected for presenting histological features of non-alcoholic steatohepatitis in liver parenchyma distant from the tumor.

The pathogenesis of $\mathrm{HCC}$ in NASH remains uncertain, although cirrhosis itself may be seen as a promoter of neoplastic transformation. However, the finding of one case of HCC in the absence of relevant fibrosis or cirrhosis shows that cirrhosis is not mandatory for HCC appearance in NASH patients. The mechanisms of pathogenesis of $\mathrm{HCC}$ in the non-fibrotic liver have not been elucidated. $\mathrm{NASH}$-associated insulin resistance causes inhibition of hepatic mitochondrial fatty acid oxidation and increased intracellular fatty acids may lead to oxidative DNA damage by stimulating microsomal peroxidases.

Another interesting aspect differed from the results

\section{References}

1. Clark JM. The epidemiology of nonalcoholic fatty liver disease in adults. J Clin Gastroenterol 2006; 40 (Suppl 1): S5-S10.

2. Brunt EM. Nonalcoholic steatohepatitis. Semin Liver Dis 2004; 24: 3-20.

3. Bugianesi E, Leone N, Vanni E, Marchesini G, Brunello F, Carucci $\mathrm{P}$, et al. Expanding the natural history of nonalcoholic steatohepatitis: from cryptogenic cirrhosis to hepatocellular carcinoma. Gastroenterology 2002; 123: 134-140.

4. Ong J, Younossi ZM, Reddy V, Price LL, Gramlich T, Mayes $\mathrm{J}$, et al. Cryptogenic cirrhosis and posttransplantation nonalcoholic fatty liver disease. Liver Transp/ 2001; 7: 797-801.

5. Caldwell SH, Oelsner DH, lezzoni JC, Hespenheide EE, Battle EH, Driscoll CJ. Cryptogenic cirrhosis: clinical characterization and risk factors for underlying disease. Hepatology 1999; 29: 664-669.

6. Bugianesi E, Vanni E, Marchesini G. NASH and the risk of cirrhosis and hepatocellular carcinoma in type 2 diabetes. Curr Diab Rep 2007; 7: 175-180.

7. Marrero JA, Fontana RJ, Su GL, Conjeevaram HS, Emick DM, Lok AS. NAFLD may be a common underlying liver disease in patients with hepatocellular carcinoma in the United States. Hepatology 2002; 36: 1349-1354.

8. Dam-Larsen S, Franzmann M, Andersen IB, Christoffersen $P$, Jensen LB, Sorensen TI, et al. Long term prognosis of fatty liver: risk of chronic liver disease and death. Gut 2004; 53: $750-755$.

9. Adams LA, Sanderson S, Lindor KD, Angulo P. The histological course of nonalcoholic fatty liver disease: a longitudinal study of 103 patients with sequential liver biopsies. J Hepatol reported by lannaccone et al. (16), i.e., most patients were classified as early stage according to the BCLC staging system and most of them were uninodular and smaller than $5 \mathrm{~cm}$. This discrepancy may be due to the fact that, in our service, patients are submitted to screening programs with serum AFP and ultrasound every 6-12 months. The non-cirrhotic patient discovered a multinodular HCC in a routine ultrasound. The fact that, although multinodular, the tumor was so well-differentiated also requires further study to search for a possible carcinogenic pathway for this intriguing case.

Cancer in our NASH patients preceded clinically advanced disease, occurred with non-diagnostic levels of AFP and can be diagnosed in the early stage in a screening program. Moreover, the association of NASH with HCC is not limited to patients with NASH-related cirrhosis. We observed $\mathrm{HCC}$ in 1 of 7 patients without cirrhosis by multiple parameters including repeated biopsy, radiographic imaging and direct surgical inspection. Further investigation of these relationships is warranted to determine relative risk and the possible need for more aggressive screening in NASH patients.

2005; 42: 132-138.

10. Calle EE, Rodriguez C, Walker-Thurmond K, Thun MJ. Overweight, obesity, and mortality from cancer in a prospectively studied cohort of U.S. adults. N Engl J Med 2003; 348: 16251638.

11. Cuadrado A, Orive A, Garcia-Suarez C, Dominguez A, Fernandez-Escalante JC, Crespo J, et al. Non-alcoholic steatohepatitis (NASH) and hepatocellular carcinoma. Obes Surg 2005; 15: 442-446.

12. Bruix J, Sherman M, Llovet JM, Beaugrand M, Lencioni R, Burroughs AK, et al. Clinical management of hepatocellular carcinoma. Conclusions of the Barcelona-2000 EASL conference. European Association for the Study of the Liver. $J$ Hepatol 2001; 35: 421-430.

13. Kleiner DE, Brunt EM, Van Natta M, Behling C, Contos MJ, Cummings OW, et al. Design and validation of a histological scoring system for nonalcoholic fatty liver disease. Hepatology 2005; 41: 1313-1321.

14. Hai S, Kubo S, Shuto T, Tanaka H, Takemura S, Yamamoto $\mathrm{T}$, et al. Hepatocellular carcinoma arising from nonalcoholic steatohepatitis: report of two cases. Surg Today 2006; 36: 390-394.

15. Shimada M, Hashimoto E, Taniai M, Hasegawa K, Okuda H, Hayashi $\mathrm{N}$, et al. Hepatocellular carcinoma in patients with non-alcoholic steatohepatitis. J Hepatol 2002; 37: 154-160.

16. lannaccone R, Piacentini F, Murakami T, Paradis V, Belghiti $\mathrm{J}$, Hori $\mathrm{M}$, et al. Hepatocellular carcinoma in patients with nonalcoholic fatty liver disease: helical CT and MR imaging findings with clinical-pathologic comparison. Radiology 2007; 243: 422-430. 